\title{
HIV/AIDS issues in the workplace of
} nurses

\author{
A Minnaar, Ph.D. \\ Senior Lecturer, Department of Nursing Education, University of the Witwatersrand
}

Correspondence address:

\section{Dr. A Minnaar}

Department of Nursing Education

School of Therapeutic Science

Faculty of Medical Science

University of the Witwatersrand

7 York Street

Parkton, 2193

Tel: 011 488-3094

Faks:

E-mail:

\section{Abstract: Curationis 28(3): $31-38$}

HIV/AIDS is a global problem with an estimated 40 million infected people. In less than two years, this figure will leap to 100 million according to the World Health Organisation (WHO). By 2005, 65 million people will be infected. Half of the number of people in this group will be under 25 years old, and will die before they reach the age of 35 . In a South African study done by the Human Science Research Council and published in 2003, regarding the impact of HIV/AIDS on the health sector, the findings were that $15 \%$ of health workers in public and private hospitals tested positive for HIV antibodies. Together with these facts above it was found that 46.2 percent of patients served in medical and paediatric wards tested positive for HIV. These factors have major implication for staffing in the future and the role of the nurse manager in South Africa. The aim of the study: to explore the management of HIV/AIDS in the workplace of nurses in selected health services in KwaZulu-Natal. This research was part of a greater study on the exploration of the presence of caring as part of nursing management.

The methodology: the qualitative research approach was used with a phenomenological design, which ensured that the richness and the complexities are reflected in the study. The data was collected by means of an open-ended question to nurse managers during an interview. The first question posed was; How do you or your services care for nurses in this hospital? Secondly nurse managers were asked,

To explain their role in caring for HIV/AIDS positive nurses on their staff establishment. The results: a qualitative analysis of the interviews with nurse managers indicated that they rate HIV/AIDS issues as an important part of their management task. Four main themes were identified, namely HIV/AIDS, counselling, dying of AIDS and funerals. Rich descriptions of these themes are given in this paper. Nurse managers in the health services are managing HIV/AIDS affected nurses, but are doing so without any formal policy on HIV/AIDS in the workplace. It is recommended that nurse managers deal with HIV/AIDS issues in the workplace by raising HIV/AIDS awareness regarding HIV/AIDS issues and HIV/AIDS in the workplace.

Conclusions: nurse managers saw their role regarding nurses with HIV/AIDS mainly as supportive - being involved in family problems and counselling and guiding nurses that live with HIV/AIDS. The health services in KwaZulu-Natal, and especially nurses, are currently affected by HIV/AIDS in a significant way.

\section{Introduction and problem}

Globally, an estimated 40 people million people are infected with HIV. In less than two years, this figure will leap to 100 million according to the World Health Organisation (WHO). By 2005,65 million people will be infected. Half of the number of people in this group will be under 25 years old, and will die before they reach the age of 35 . Approximately one in very four sexually active persons in SA is infected. South Africa is believed to be the country with the fastest growing HIV epidemic in the world, with an estimated 2000 new cases every day. In a South African study done by the Human Science Research Council and published in 2003, regarding the impact of HIV/AIDS on the health sector, the findings were significant in that $15 \%$ of health workers in public and private hospitals tested positive for HIV antibodies. Furthermore, the study found 
that 46.2 percent of patients served in medical and paediatric wards tested positive for HIV. (HSRC 2003:131-136)

It is estimated that HIV has been around for longer than 30 and less than 100 years. In Africa it is known as "Slims". According to speculation it originated in Central Africa and spread to humans via the Green or Simian Monkey. Simian monkeys carry a similar retrovirus (reversed flow of genetic information from RNA to DNA), called SIV. In 2000, AIDS deaths totalled three million worldwide. Of these deaths, 2.4 million occurred in Africa. The fight against HIV transmission is a challenge from which the world cannot cower. The HIV/AIDS threat continues to grow. As AIDS advances further into its third decade. the question that faces the global community is whether we shall have the commitment to respond effectively. Will our expressions of concern be turned into concrete action? Having failed to prevent the first wave of the epidemic, shall we have the courage and vision to prevent the next one? (Walt \& Gilson 1994:353-370)

It is important to note that an internal HIV/AIDS workplace response is simply one aspect of a broader, integrated HIV/ AIDS strategy, which includes a range of external responses to HIV/AIDS. Internally, the Health Service must respond as an employer, recognising the impact on both infected and affected employees, and patients. Externally, the Health Service is a sector, which, along with the private sector and civil society, must act collectively to address the epidemic

HIV/AIDS is never far from making the headlines. The scenario that was created at the Nursing 2000 Conference in Midrand, which was held during the first week in September 2000, indicated that $20 \%$ of the South African nursing population could theoretically be HIV positive. That means that 34000 of the 174000 nurses that are available for duty in South Africa, could be HIV positive. and that one student nurse would die of AIDS every month (Geyer, 2000). The Gauteng Department of Health indicated that no proof exists for these statistics, and that it is not clear how these figures were leaked. Questions are being raised regarding its accuracy.

AIDS is not a disease; it is a development disaster (Gayle \& Gates 2003:5). See Figure 1 for statistics on HIV/AIDS infected people globally. The abbreviated term HIV+ refers to human immunodeficiency and infected and infectious, and AIDS refers to HIV and severe immune dysfunction and AIDS-defining illness.

This study aimed to explore HIV/AIDS in the workplace of nurses and forms part of a greater study on caring in nursing management in KwaZulu-Natal. The phenomenological design was utilised for this qualitative research approach to explore the meaning and lived experiences of nurse managers regarding the management of HIV/AIDS affected nurses in the workplace in Health care organisations in Kwa-Zulu Natal. The qualitative approach was chosen to help the researcher understand the experiences of nurse managers regarding HIV/AIDS among nurses in the context in which they function and live every day. The goal of the qualitative approach to the study was to bring about understanding of the impact of HIV/AIDS among nurses from the perspective of nurse managers.

In South Africa, the Health Service is the umbrella organisation, of which nurses form a large percentage of the workforce. Although it remains difficult to predict the extent of the future impact of HIV/ AIDS on the Health Service, it is clear that departments are already, and will increasingly be, affected. The problem is that nursing service delivery will be negatively affected - not only due to the number of infected employees, but also due to the increased demand for certain health services. What are the issues which nurse managers need to address regarding HIV/AIDS in the workplace of nurses?

Health Services in remote areas and disadvantaged communities will be particularly vulnerable to absenteeism and deaths that result from HIV/AIDS due to the shortage of skilled nursing and other staff, as well as resource constraints. HIV/AIDS will increase the need for the training of replacement staff, while at the same time compromising the potential for mentoring and skills transfer. Sick leave could increase dramatically in all services, with devastating effects on the nursing care delivery systems of health services.

Clearly a coordinated and effective response is required to minimise the impact of HIV/AIDS on the Health Service, because unless managed, the impact of HIV/AIDS will make it difficult to achieve the transformation goals of government. An integrated HIV/AIDS response will promote confidence and will increase morale in the Health Service, with spin-offs that will benefit the communities at large. Such a response will also allow the Health Service to demonstrate its commitment to fairness, equity and compassion (Barrett-Grant, Strode \& Smart 2002:32). This research was therefore undertaken to explore HIV/ AIDS and its effect on nurses from a management perspective.

\section{Purpose and research objectives}

The purpose of the study was to explore HIV/AIDS in the workplace of nurses. The specific objectives for the study were to
a)
explore HIV/AIDS in the workplace of nurses from a management perspective, and
b) recommend HIV/AIDS guidelines for nurse managers according to which they can handle HIV/AIDS in the workplace.

\section{Conceptual framework}

Any workplace response to HIV/AIDS must be based on an understanding of the rights of persons that are infected and affected by HIV/AIDS. A number of important international guidelines were developed to guide the response of governments and its departments to HIV/ AIDS. The UNAIDS and Human Rights International Guidelines (1998), as described by Barrett-Grant, Strode, \& Smart (2002:1-10), are international guidelines to assist in creating a positive, rights-based response to HIV/AIDS, which is effective in reducing the transmission of HIV and the impact of the epidemic, and which is consistent with human rights and fundamental freedom (Davies, Scheider, Rapholo and Everatt 1997:1). The SADC Code of Good Practice on HIV/AIDS and Employment (1997) was developed through a consultative tripartite process and adopted at a meeting of Ministers of Labour in Pretoria, South Africa in August 1997. Although the Code is not a legally binding document, all those who were signatories to it agreed that the national and regional implications of the 


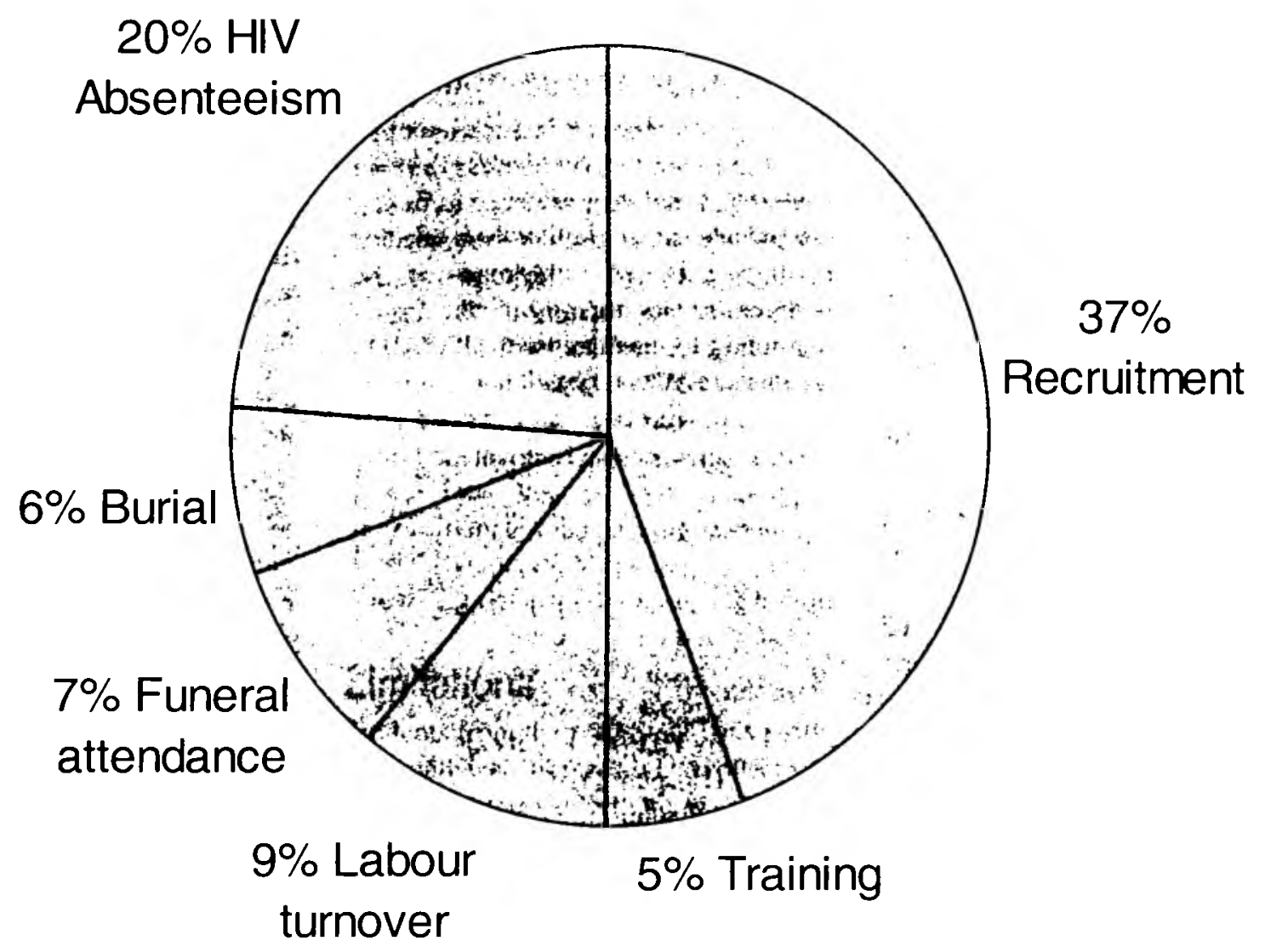

HIV/AIDS epidemic meant that a need exists to have regional employment standards, and that all member countries should develop tripartite national codes that are reflected in national law (Davies, Scheider, Rapholo and Everatt 1997:3). The International Labour Organisation (ILO) Code of Practice on HIV/AIDS and the World of Work (2001) bind all employers and employees in the private and public sector and all aspects of work. formal and informal. Standards that can be used in developing a response to HIV/ AIDS in the workplace are set out in the ILO Code, as described by Barrett-Grant, Strode, \& Smart (2002:1-10) in the following way:
a) HIV/AIDS must be recognised as a workplace issue;
b) responses to HIV/AIDS must be based on the principle of non-discrimination:
c) gender equality must be pursued as part of any HIV/ AIDS response;
d) every employee has a right to a healthy and safe working environment;
e) social dialogue between employers, workers, their representatives and

government must take place regarding HIV/AIDS issues;

f) there must be no HIV screening of job applicants or employees;

g) every employee has the right to confidentiality regarding his/ her HIV status;

h) workers must be enabled to continue working for as long as possible;

i) workplaces must promote HIV prevention; and

j) care and support should be provided to infected workers (Barrett-Grant, Strode, \& Smart 2002:1-10).

Furthermore, the Employment Equity Act, Act No. 55 of 1998, which aims at ensuring equality and non-discrimination in the workplace through anti-discrimination measures and affirmative action provisions, has a bearing on HIV/AIDS in the workplace. Two clauses in the Act expressly refer to HIV/AIDS, namely the prohibition on unfair discrimination based on "HIV status", and HIV testing without Labour Court authorisation (Dye 1995:10).

A coordinated and effective response to
HIV/AIDS in the nursing workplace should start with sufficient knowledge of HIV/AIDS issues among all levels of personnel. The nurse manager could do a survey to establish the level of knowledge and beliefs regarding HIV/ AIDS among nursing staff, as well as among other staff members in the health service. Basic knowledge of the disease comprises knowledge of the five stages in which it presents itself.

Firstly, the primary HIV infection (window period lasts up to three months) takes place within a few weeks after incipient HIV infection, and it is at this time that people change from HIV negative to HIV positive. This stage lasts for only a few weeks, and the person looks and feels completely well. Some people may develop a tlu-like illness with fever, sore throat, swollen glands, headache, muscle aches and skin rash (Davies. Scheider, Rapholo and Everatt 1997:5).

Secondly, the asymptomatic or silent stage, during which the person looks good for approx 4-11 years. Having recovered from the initial infection, the person is now completely well for a long period of time, often many years. This 
means that the person feels healthy and can easily infect other people through unprotected sex and blood. In the meantime the HIV still continues to destroy the immune system. (Davies, Scheider, Rapholo and Everatt 1997:5).

Then, thirdly, several years after infection, follows the early HIV symptomatic disease (immune suppression, which lasts for (wo years). Some people will begin to show mild symptoms of the HIV disease, which could include shingles, swollen lymph glands, fevers, skin irritations, fungal infections and nail infections, e.g. oral thrush, oesophageal invasion of thrush, common dermatophyte infections including tinea pedis and tinea cruris, cryptococcus meningitis, chest infections such as pneumocystis carinii (pneumonia) with sub-acute symptoms such as fever, dry cough, fatigue, dyspnoea and pain with inspiration and lastly weight loss (Davies, Scheider, Rapholo and Everatt 1997:5).

The medium-stage of HIV, with a CD4 count of 200 to 300 , follows fourthly, and is known as the "AIDS-related complex". This is when the person with AIDS becomes very ill. Typical problems might include tuberculosis, recurrent fungal infections and herpes blisters on the mouth (cold sores), ongoing fever, persistent diarrhoea and weight loss (loss more than 10\%) (Davies, Scheider, Rapholo and Everatt 1997:5).

Lastly. the late stage of HIV is the symptomatic disease stage called "AIDS", which lasts more or less two years, with a CD4 count of less than 200 . Long-term damage to the immune system causes severe opportunistic infections, cancers and other damages, i.e. to the brain. Opportunistic infections include severe diarrhoea, severe weight loss, severe pneumonia, bronchitis, meningitis, dementia, blindness, cancer in the form of Kaposi Sarcoma, skin rashes and pain (Davies, Scheider, Rapholo and Everatt 1997:5).

Ultimately, policies, broad principles and guidelines constitute the groundwork for the intervention programmes. Nurse managers need to plan a strategy for the intervention programmes, and sufficient resources need to be available to implement the programmes. A workplace HIV/AIDS programme needs to be drawn up in consultation with all relevant stakeholders in the health services. Training programmes, support and counseling form part of this intervention. (Freeman 2003:1-30).

\section{Population and sampling}

The target population included general provincial and private hospitals in KwaZulu-Natal. Convenience sampling applied where the hospitals were selected according to the judgement of the researcher in terms of the most representative hospitals with HIV/AIDS issues and HIV/AIDS patients. Hospitals were approached and asked for permission to do the research, and only hospitals that agreed and gave permission were included in this research (Polit and Hungler 1993:179-180). Three hospitals in KwaZulu-Natal were included in the study, namely

a) a medium size non-government city hospital with medical wards for HIV/AIDS patients

(Hospital 1),

b) a medium size private mission hospital with medical wards for the caring of HIV/AIDS

patients, which is known for its work and research on HIV/AIDS (Hospital 2), and

c) a medium sized general provincial hospital in a more rural area with large numbers of HIV/AIDS patients (Hospital 3).

\section{Research methodology}

The principles of phenomenological research design were applied to explore the meaning of HIV/AIDS to nurse managers in the workplace. Individual interviews were held with nurse managers with the following posed questions, firstly;

How' do you or your services care for the nurses in this hospital?

Secondly;

Explain your role in caring for HIV/AIDS affected nurses on your staff establishment.

The participants were informed that their experiences should focus on their current positions. Each participant was assigned a code number according to the hospital, as an assurance of confidentiality, e.g. (1.1). The participants were asked by the researcher to identify a suitable venue, date and time for the interviews between the researcher and nurse managers. Nurse managers and middle managers were interviewed individually for 20 - 80 minutes. Nursing service managers were approached, and the participants were asked to volunteer for participation in the study. The data collection was terminated as soon as the data was saturated The data was audio taped, with the consent of the participants. A total of twelve nurse managers from the three hospitals were interviewed. (Oiler 1986:69-84).

The researcher aimed at capturing the rich descriptions of nurse managers regarding their everyday experiences with HIV/AIDS affected nurses and data was collected in a contextually informed way by capturing not just the words, but also the differences in pitch, gestures, body language, silences and simultaneous speech in two ways. Firstly, the researcher took notes during the interviews. Secondly, the researcher audio taped the interviews with the permission of the participants. Lastly, the researcher captured the data by using the information on the tapes together with the researcher's notes to transcribe each interview into text. This was done soon after the interview to ensure that a complete picture of each interview was captured by the researcher. The raw data, as recorded, was transcribed for each participant. Before working with the data, the researcher needed to identify her own preconceptions regarding nurses affected by HIV/AIDS. The researcher's own preconceptions needed to be set aside (the process of bracketing). That enabled the researcher to be more open and to validate her own beliefs and concerns while recognising that biases cannot be controlled fully.

Also, the data was analysed qualitatively with the help of the computer software program called NUD*IST 4 . The meaning of lived experiences of nurse managers was explored in this study. The interviews and the observations during the interviews were made in natural settings. The first step comprised the collection of lived descriptions and experiences of participants. The data came in the form of audio taped interviews with participants, and the researcher's field notes. The audiotapes were transcribed and typed using a word processing computer programme. The transcripts in its entirety were read over and over. The researcher interacted with the data through highlighting sections of the transcript by using the computer programme NUD*IST 4 (Mackey 2005: 179-184). With the onset of data analysis, a preliminary understanding of the 
meaning of HIV/AIDS in the workplace of nurses was already formed. The issue of nurses affected by HIV/AIDS emerged from the first interview, (total of twelve interviews) and it was clear that nurse managers spend much of their time dealing with HIV/AIDS issues affecting nurses. The second step comprised the reading and rereading of the descriptions of the participants on the phenomena of HIV/AIDS in the workplace of nurses. During this step, the data was explored for deeper regularities and irregularities, as well as for differences and similarities. Aspects and descriptions that really stands out from the data were highlighted. Units with the same meaning or idea were identified. The third step in the data analysis comprised the extracting of significant phrases and statements from the manuscripts (Terre Blanche and Durrheim, 1999:101). Key statements of participants that have a bearing on nurses affected by HIV/AIDS were identified and, after careful thought, free nodes were created by using the NU*DIST 4 computer programme.

After identifying the key phrases and statements, the fourth step was to identify and name themes from the identified statements. It was done by the bottom-up approach, as described by Terre Blanche and Durrheim (1999:102). It was used to organise the data that naturally underlies the material. Identifying the essential structure of the phenomenon of HIV/AIDS in the workplace of nurses involves taking all the themes and trying to identify the commonness or essential structure of HIV/AIDS affected nurses in each transcript. The rich description of everyday living and caring for HIV/AIDS affected nurses was shared with the participants, and the participants verified the data as a true reflection of what was said during the interviews (Gillis \& Jackson 2002:191-192). It was also important to spend enough time with the participants to get a clear understanding of the experiences of nurse managers regarding caring for HIV/AIDS affected nurses in the workplace. The major themes that were extracted from the data were HIV/AIDS, counselling, dying of AIDS and funerals and support.

The fifth step comprised the verification of the essential structures of HIV/AIDS in the workplace of nurses, as identified by the data analysis, in collaboration with the participants. It was done by means of supplying the participants with a written version of the phenomenon and asking them to reflect on the identified structures for HIV/AIDS in the workplace of nurses. The sixth and last step comprised the studying of the interpretation of the phenomenon by the participants after their reflection on the structures for HIV/AIDS, and to carefully identify weak points and contradictions in the interpretations. This was very timeconsuming, and the objectiveness of the researcher during this step was of the utmost importance (Terre Blanche and Durrheim, 1999:104).

\section{Ethical considerations}

Written permission was obtained from Department of Health, the selected hospitals and from the participants. Informed consent was sought from the participants. The health services were not identified at any stage of the research. Participants were ensured that they could withdraw at any stage without any discrimination. No risks for the participants could be identified. Permission was asked form the participants to take notes and to use a tape recorder during the interviews. Respect for privacy of the participants was honoured and confidentiality of information was maintained by the fact that no names were revealed during the research study or in the research report or any publications of the research.

\section{Trustworthiness}

The trustworthiness of the study was demonstrated by the fact that the study was done over a period of three years, and that the data was collected from the nurse managers in their own environment. The documented and transcribed evidence from the interviews was taken back to the participants, who confirmed that the transcription was a true reflection of what was said during the interviews. The themes were taken back to the participants for verification. (Poggenpoel 2000:349-350).

\section{Results}

The results are discussed under the headings of the themes that emerged from the data. Direct quotes from the interviews are typed in italics and are indented in the text. Reference to a specific quote indicates the hospital and the transcript line number, e.g. $(1,102)$. The researcher added the italic text, which is bracketed, in order to create better understanding, e.g. ... (HIV/AIDS is visible) ... In some cases, in order to clarify meaning, a word or words might have been altered, which is indicated by square brackets around the alteration, e.g. [relieved].

This research emphasised the importance of an internal HIV/AIDS workplace response in the health services. It also seemed important to look at the competencies that nurse managers should have to enable them to deal with the complex problems of this killer disease, as well as with the demands that it puts on nursing resources. Support to the families of nurses with the disease seemed to be a major issue because of the influence it has on health services and the nurses themselves. Nurse managers saw their role, to a great extent, as dealing with the disease and supporting the families of nurses. Nurse managers emphasised their commitment to their staff by supporting the staff members in dealing with the disease, and organising the funerals of staff members who died of AIDS.

\section{Theme 1 - HIV/AIDS}

HIV/AIDS among nurses and the problems that are associated with it seemed to take up a lot of the nurse managers' time. An example, as described by a nurse manager, is given below, illustrated the amount of time spend on nurses affected by HIV/AIDS. One nurse manager reflected her involvement with a nurse diagnosed with AIDS. From the description below, it is clear that nurse managers go beyond their conventional management duties when their nurses are affected by HIV/AIDS.

And then I want to quote an incident where a member of staff with a needle stick injury. In the hospital she contacted HIV. We went through the incident with her and she was sick for quite a long time, I was very supportive because when she was very, very ill, I made the point of visiting her at home. We took the hospital vehicle to take her home, when she was admitted to hospital and got discharged. And when she was sick at home I did not want her family to bring her here. I always communicate with her family. If they needed the vehicle they must not hire one. I used to go and fetch her myself or send a driver if the driver was not available I would go there myself or Mrs $M$ would go. When she was admitted at the hospital, there was a strike, Mrs M 
drove because it was so difficult to get into the hospital, so she drove herself and went to the hospital in her own car because the hospital car was not available that day, to bring her back to our institution and we nursed her here. (When she died) I communicated with her mother, because she was a breadwinner, and I asked her mother if there was a sister who was actually big enough to get employment at the hospital. I discussed it with the management. and we all agreed that we should offer this family assistance, because they were a very, very poor family. So I asked the mother to bring the sister here, I interviewed the sister and I employed her as a general assistant. She was qualified and did a secretary course after her matric. She accepted the post as an assistant. I did not have a post for a secretary at that time, with the hope when there is a post in admin. she can apply for it. Unfortunately she also contracted HIV and she died. she died two months ago (3.345).

\section{Theme 2 - Counselling}

Counselling was described in terms of counselling nurses diagnosed with HIV/ AIDS. The nurse managers saw the counselling of nurses as a major part of their management functions. In some of the hospitals. the need for a person to counsel the staff on HIV/AIDS issues was so great that a nurse counsellor was appointed. The excerpts below describe this scenario.

We do AIDS counselling. I myself am an AIDS counsellor. I do know some of the staff is HIV positive and in the allocation list. (I do the allocation). I try to place them in suitable departments. For a lot of them. I have torn up the HIV results and keep it confidential. I think also in the office here. confidentiality plays a very important role (3.207-212).

We have a pastoral counselling service that is caring for the nurses. We got a senior counsellor. one of the senior professional nurses is the counsellor. She visit them in the wards, she talks to them spiritually $(2,39-41)$.

Also at the same time. we have a counsellor for HIV/AIDS. At present we are looking for someone for counselling the staff. She comes from the first of October. She will look at counselling the AIDS stricken staff members. The staff actually has family members that are HIV/
AIDS positive. They themselves might also not be feeling well, and then somebody, not the matron or a colleague (need to be available to them) to open up to, someone who is neutral, so provision is being made for this $(2,254-260)$.

If it needs a doctor to intervene, we get somebody to intervene. If you find that you cannot intervene yourself, you could always find somebody to intervene so that the work flows smoothly. That is why you are always there, and they are free to report to us anytime. (long silence) There are times when we have deaths and we feel our staff is stressed out even with that. we asked our counsellor to counsel them, to come to the group especially to our medical ward. The councillor is supporting the affected (long silence) (1.203).

Because with the elderly person, it is much better. but with the young ones... Now you can see it is so difficult. almost every day and we liaise with our counselling department. We got a counselling department $(1,445)$.

\section{Theme 3 - Dying of AIDS}

Employees who have HIV/AIDS were managed well by nurse managers in the health services. Employees that are living with HIV/AIDS can be productive for a long period if they receive the medical, social and psychological support they need. It is important to develop guidelines and policies according to which managers can assess and manage the situation (Davies. Schneider, Rapholo and Everatt, 1997). Taking care of dying staff was very prominent at the hospitals. and was expressed as excerpted below.

With the few resources they always have a complaint. we are overworked, because they (the nurses) are sick. They don't come on duty: they stay off sick. put in leave again and again because their immune system has gone down. Flu, so many [of the nurses] have flu they stay away with flu for two months. No doctor's certificate is presented, that is a problem $(1,43-48)$.

One of the things that came up, when we were discussing strategic planning, is the handling of dying patients. This was brought up because most of our nurses especially in the medical wards are faced with death. Especially with HIV and AIDS (1.548).

Nursing is down because the nurses themselves are sick, they are sick with HIV and in all these wards the patients (12 a day die), gone dead, they are dying. that is so demoralising and they even said you know they are going to die and even themselves (the nurses) they die by HIV/ $\operatorname{AIDS}(1,33-36)$.

\section{Theme 4 - Funerals}

Funerals that are related to HIV/AIDS were a prominent issue at the hospitals, and it was expressed as excerpted below.

They want the matron to visit them at the hospital and they (the family of the nurse) want the management to be there at the nurse's funeral and to take a lead in organising the nurse's funeral, especially the African nurses. The other races are not a problem, but the African nurses want the management to get involved in organising the funerals for a nurse who dies, and the management to be there at the funerals (of nurses) (3, 105-110).

I told you about development. sick leave, involvement in organising the funerals of nurses, they really want us to do that, so what I did is, I ask the nurses to form their own organisation in the Hospital, which will support them. the funeral group scheme and they contribute every month to it $(3.442)$.

The fact that nurse managers spend a lot of their time on HIV/AIDS-related aspects could be an indication of the seriousness of the infection rate and the effect of the disease on nursing services. Nurse managers indicated that they spend a great deal of their time on counselling nurses with HIV/AIDS, taking care of the ill nurses and organising funerals of nurses who have died of AIDS and related diseases.

\section{Discussion}

Nurse managers saw their role regarding nurses with HIV/AIDS mainly as supportive: being involved in family problems and counselling and guiding nurses that are living with HIV/AIDS. Nurse managers were involved in planning and organising the funerals of nurses who died from HIV/AIDS. The nurse managers seemed to play a major role in counselling the nurses that are suffering from HIV/AIDS in the health services. Nurses needed counselling not only because they contracted the disease. but also to help them cope with the changing demands of the high number 
of patients who no longer recover from the illnesses, but die as a result of AIDS.

Nurse managers saw the physical welfare of nurses as very important. It was clear from this study that nurse managers were mainly concerned about the physical well-being of nurses. Support to nurses in dealing with HIV/AIDS issues was addressed, but the implementation of an HIV/AIDS policy was not in place in the health services. Professional, userfriendly and confidential services need to be established in the workplace, for both the employee and employer, and in such a manner that nurses can access it in an effortless way. Counselling nurses that are affected with HIV/AIDS should not only include information, but should enable the person to choose between various options while providing a psychological and emotional supportive relationship.

Health services should be at the forefront in managing HIV/AIDS in the workplace by starting to render primary health services on site. Counselling and support services for nurses and other categories of employees that are affected with HIV/ AIDS should be in place to ensure that employees stay in the services as long as possible, in order to lessen the financial burden on the remaining workforce. See Figure 1 for increased costs due to the impact of HIV/AIDS on the workplace in business in South Africa. The estimations were done by Freeman (2003) and it indicates that re-training of staff increased by $5 \%$. Staff members in business in South Africa needed to be re-trained as a result of staff loses or staff member who died as a result of AIDS.

Staff turnover increased by $9 \%$ as a result of HIV/AIDS among workers in South Africa. Staff turnover contribute to staff shortages and in hospitals the problem is double because of patient increases and more patients with HIV/ AIDS related diseases. Work increases lead to absenteeism among the remaining staff and organisations experienced chronic staff shortages. According to Figure 1 costs due to absenteeism is up by $20 \%$. The alarming fact is that recruitment of personnel cost $37 \%$ more according to figure 1 and this hamper the quality of services in South Africa to a great extend.

Lastly the time and costs of funerals increased by $7 \%$ and burial by $6 \%$ due to
HIV/AIDS (Freeman 2003:52). Furthermore, the important role of nurse managers regarding the organisations and attendance of funerals when nurses die of HIV/AIDS is clearly illustrated in this study. The costs of this factor in nursing is not yet established.

\section{Recommendations and conclusions}

A recommendation is to do further research on the effect of HIV/AIDS on the health delivery systems, in order to establish the needs of nurse managers regarding support, guidance, counselling and care to dying nurses. The influence of HIV/AIDS-positive nurses with their specific needs from nurse managers in hospitals should be estimated, and additional support systems should be put in place. The ability of nurses living with HIV/AIDS should be established, and their capabilities to render nursing care should be investigated to enable nurse managers to supply enough nurses to render quality nursing service. Sufficient additional support and guidance to nurse managers and nurses should be provided to cope with the demands of HIV/AIDS positive nurses.

A strategy for mainstreaming HIV/AIDS in the workplace of nurses should receive the urgent attention of nurse managers in hospitals, as well as nurse educators in the whole of South Africa. Leadership and management commitment to HIV/ AIDS in the health services need to comprise three components. Nurse managers need to demonstrate leadership in handling HIV/AIDS issues, and care towards nurses that are affected by HIV/AIDS in the health services. Externally, health services should lead other stakeholders in responding to HIV/ AIDS in the workplace. Personally nurse managers must act as personal role models by not discriminating against people that are living with AIDS (PLWAs) (Heard 2003: 1- 10).

Furthermore, nurse managers could deal with HIV/AIDS issues in the workplace by raising HIV/AIDS awareness at trade union meetings and by ensuring that trade unions place leadership regarding HIV/AIDS on the bargaining agenda. Nurse managers should be requested to clearly establish HIV/AIDS support services for HIV/AIDS affected nurses. A permanent HIV/AIDS slot on the staff meeting agenda should be requested to discuss the progress reports on HIV/
AIDS workplace issues. (Barrett-Grant. Strode, \& Smart 2002:1-20).

Nurse managers need specialised skills in dealing with the impact of HIV/AIDS on nurses and health services. Therefore the decisions to train nurse managers and to upgrade knowledge on HIV/AIDS management in the workplace, together with the implementation of the policy on HIV/AIDS in the workplace, need to be the highest priority for the health services. Awareness programmes should be a priority for nurse managers, as well as for those in all the public services in South Africa. It is estimated that by 2010 , $15 \%$ of highly skilled employees will have contracted HIV (Freeman 2003 52). What will happen to staff morale and skills in the health services?

\section{References}

BARRETT-GRANT, K; FINE, D; HEYWOOD, M \& STRODE, A 2001: HIV/AIDS and the Law. Cape Town: The AIDS Law Project and the AIDS Legal Network.

BARRETT-GRANT, K; STRODE, A \& SMART, R 2002: Managing HIV/AIDS in the workplace a guide for Government Departments, Pretoria: The Department of Health Service and Administration.

DAVIES, S; SCHNEIDER, M; RAPHOLO, G \& EVERATT, D 1997: Guidelines for developing a workplace policy and programme on HIV/AIDS and STIs. Department of Health

DYE, TR 1995: Understanding Public Policy. Englewood, N.J.: Prentice Hall

FREEMAN, J 2003: Toolkit: Managing HIV/AIDS in the workplace. Services Seta.

GAYLE \& GATES 2003: Global mobilization for HIV prevention a blueprint for action. Global HIV prevention working group.

GEYER, N 2000: AIDS statistics in perspective. Nursing Update. p 1 (col. 2).

GILLIS, A \& JACKSON, W 2002: Research for nurses: Methods and interpretation. Philadelphia:F.A. Davis Company.

HEARD 2003: "HIV/AIDS Mainstreaming: a definition, some 
experiences and strategies". HEARD.

HUMAN SCIENCE RESEARCH COUNCIL (HSRC) 2003: Study No. 2 The impact of HIV/AIDS on health workers employed in the health sector.<http:www.hsrcpublishers.ac.za> (Accessed 15.2.1005)

MACKEY, S 2005: Phenomenological nursing research: methodological insights derived from Heidegger's interpretative phenomenology. International journal of Nursing studies. 42: 179-184.

OILER, CJ 1986: Phenomenology: The method. In P. Munhall and C.J. Oiler (Ed.), Nursing research A Qualitative Perspective (pp69-84). Norwalk: Appleton-Century-Crofts.

POGGENPOEL, M 2000: Data analysis in Qualitative research. In A. S. de Vos (Ed.), Research at grass roots a primer for the caring professions (pp334-353). Pretoria: Van Schaik Publishers.

POLIT, PF \& HUNGLER, BP 1991: Nursing Research: Principles and methods. 4th edition. Philadelphia: Lippencott.

TERRE BLANCHE, M \& DURRHEIM, K 1999: Research in Practice Applied methods for the social sciences. Cape Town: UCT Press

WALT, G \& GILSON, L 1994: "Reforming the Health Sector in Developing Countries: The Central Role of Policy Analysis". Health Policy Planning. Vol. 9 (4): 353-370. Oxford University Press: Oxford. 